\title{
Sub-Angstrom oscillation amplitude non-contact atomic force microscopy for lateral force gradient measurement
}

\author{
Mehrdad Atabak $^{\mathrm{a}, 1}$, Özhan Ünverdi ${ }^{\mathrm{b}}, \mathrm{H}$. Özgür Özer ${ }^{\mathrm{c}}$, Ahmet Oral ${ }^{\mathrm{b}, *}$ \\ ${ }^{a}$ Department of Physics, Bilkent University, 06800 Bilkent, Ankara, Turkey \\ ${ }^{\mathrm{b}}$ Faculty of Engineering \& Natural Sciences, Sabanci University, Tuzla, 34956 Istanbul, Turkey \\ ' Department of Physics, Faculty of Science and Letters, Istanbul Technical University, Maslak, 34469 Istanbul, Turkey
}

\section{A R T I C L E IN F O}

\section{Article history:}

Available online 20 October 2009

\section{Keywords:}

Non-contact lateral atomic force microscopy

Small oscillation amplitude

Lateral force gradient-distance

spectroscopy

\begin{abstract}
A B S T R A C T
We report the first results from novel sub-Angstrom oscillation amplitude non-contact atomic force microscopy developed for lateral force gradient measurements. Quantitative lateral force gradients between a tungsten tip and $\operatorname{Si}\left(\begin{array}{lll}1 & 1 & 1\end{array}\right)-(7 \times 7)$ surface can be measured using this microscope. Simultaneous lateral force gradient and scanning tunnelling microscope images of single and multi atomic steps are obtained. In our measurement, tunnel current is used as feedback. The lateral stiffness contrast has been observed to be $2.5 \mathrm{~N} / \mathrm{m}$ at single atomic step, in contrast to $13 \mathrm{~N} / \mathrm{m}$ at multi atomic step on $\mathrm{Si}\left(\begin{array}{lll}1 & 1 & 1\end{array}\right)$ surface. We also carried out a series of lateral stiffness-distance spectroscopy. We observed lateral stiffness-distance curves exhibit sharp increase in the stiffness as the sample is approached towards the surface. We usually observed positive stiffness and sometimes going into slightly negative region.
\end{abstract}

(c) 2009 Elsevier B.V. All rights reserved.

\section{Introduction}

Lateral forces play an important role in variety of phenomena in our daily lives as well as micro and nanoscale systems. Initial approaches to understand the origin of the lateral forces associated with the relative motion of two objects were mainly based on collective mechanical properties of interacting objects [1]. However, investigation of lateral forces and interaction energy at the molecule and atomic scale needs accurate measurement of lateral stiffness of single chemical bonds established between the objects in contact. Lateral force microscopy (LFM) has shown its capability to image and identify lateral forces at the atomic scale while a sharp tip scans over a sample surface [2].

In 1987, Mate et al. [2] imaged lateral forces acting between a sharp tungsten tip and a graphite surface and the stick-slip behaviour was observed. Later on, different research groups [3,4] detected atomic stick-slip behaviour using a UHV-FFM (frictional force microscopy)apparatus on ionic crystals and metal surfaces and compared with a theoretically produced lateral force maps based on two-dimensional Tomlinson model. Studies of atomic scale origins of lateral forces while the sharp tip is scanned in low load contact over sample surfaces have already contributed to the understanding

\footnotetext{
* Corresponding author. Tel.: +90 216483 9522; fax: +90 2164839550.

E-mail address: aoral@sabanciuniv.edu (A. Oral).

1 Present Address: Omicron Nanotechnology GmbH, Limburger Str. 75, 65232 Taunusstein, Germany.
}

of the microscopic origins of friction forces. However, the lateral resolution of force microscopy in contact mode is limited by the contact area of the tip apex, containing many atoms due to adhesion between tip and sample. This problem has been eliminated in noncontact atomic force microscopy. Jarvis et al. [5] developed a special cantilever for simultaneous control of tip-sample distance and lateral tip oscillations, in order to sense lateral interactions between tip and sample when approaching the surface. Giessibl et al. [6] presented atomic resolution in lateral forces, while using a tuning fork as a tilted lateral force sensor. Their sensor had a glued tungsten tip at the end of one prong of the tuning fork, while the other prong is fixed. The tip was intentionally tilted with respect to the sample by $6^{\circ}$. They showed that the interaction between a single-tip atom that is oscillating with $3 \AA$ slightly canted to the $\operatorname{Si}\left(\begin{array}{lll}1 & 1 & 1\end{array}\right)-(7 \times 7)$ surface can be measured in dynamic mode AFM. However, the tilt of the force sensor with respect to the sample might reflect the contribution of normal forces gradient in addition to lateral force component. In another work, Pfeiffer et al. [7] reported measurement of lateral forces between the tip of a force microscope and the atomic-scale features on the surface in a non-contact mode using $20 \AA$ oscillation amplitudes. In their experiment, a rectangular cantilever beam oscillates parallel to the $\mathrm{Cu}\left(\begin{array}{lll}1 & 1 & 1\end{array}\right)$ sample surface at its torsional Eigen mode, while the tunnel current is kept constant with the feedback loop.

In a recent work, Kawai et al. [8] also reported atomic resolution in lateral force using few Angstrom amplitude with the frequency modulation dynamic lateral force microscopy. In their experiment, torsional resonance mode of a commercially available rectangular 
Silicon cantilever was used to detect the lateral interaction force gradients caused between the tip and the sample surface. Schwarz et al. [9] has shown three dimensional force field spectroscopy method and demonstrated interatomic vertical and lateral forces

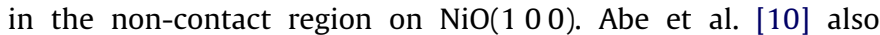
calculated the interatomic potential and the lateral force mapping from the measurement of frequency shift curves in vertical direction using FM-AFM. Sugimoto et al. [11] numerically deducted the frequency shift data and surface potential map using an experimentally obtained $F_{z}$ measurement and demonstrated the enhancement of topographic corrugation with the decrease in the oscillation amplitude. In this work, the lateral force numerically deduced from the calculated potential map.

All of the above attempts have shown that the sub-Angstrom oscillation amplitude lateral force microscopy would reveal more insight into the lateral force interactions between tip and sample, down to single atom. In this report, we describe the sub-Angstrom oscillation amplitude non-contact atomic force microscope for lateral force measurements and present the first results from the microscope. Simultaneous lateral stiffness and STM topography images on $\mathrm{Si}\left(\begin{array}{lll}1 & 1 & 1\end{array}\right)-\left(\begin{array}{l}7 \times 7 \\ 7\end{array}\right)$ surface are obtained. We also present the direct measurement of lateral forces as we vary the tip-sample separation, similar to our earlier experiments using ultra small amplitude non-contact AFM/STM [12-15] in normal forces.

\section{Experimental methods}

A home-made, high resolution non-contact atomic force microscope/scanning tunnelling microscope operating in UHV is used in our experiments. The microscope is designed to detect normal and lateral forces simultaneously with the tunnel current, however we concentrate only on the lateral force measurements for the moment. The microscope is mounted on a special $8^{\prime \prime} \mathrm{CF}$ flange as shown in Fig. 1. The microscope base plate is suspended using four stainless springs on four posts attached to the flange. Four Sm-Co magnets fixed on a ring is attached to the posts and copper plates attached to the microscope base plate, these serve as the vibration damper. Electrical connections are achieved using Teflon and kapton insulated wires and two 20-pin electrical feedthroughs mounted at the microscope flange. A $25.4 \mathrm{~mm}$ long, $12.7 \mathrm{~mm}$ diameter and $1 \mathrm{~mm}$ thick EBL\#2 piezo tube with four outer electrodes is used for the sample scanner. The sample scanner is shielded and then mounted on a shear-piezo driven $X Y \Phi$ axis nanopositioner for coarse approach as shown in Fig. 1. A $10^{8}$

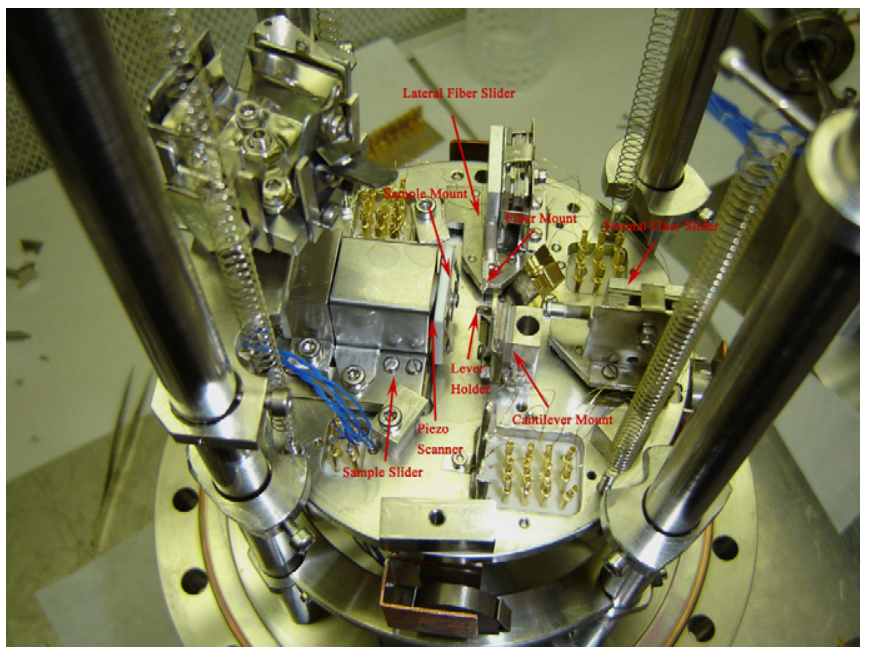

Fig. 1. A picture of the non-contact atomic force microscope for simultaneous lateral and normal force measurements. gain $i-v$ converter is placed just behind the scanner tube and connected to the sample. A 2-in. travel linear motion feedthrough concentrically mounted on the microscope flange is used to lock the microscope base plate and the sample slider during cantilever and sample exchange. Cantilevers and samples are mounted on special stainless steel and Molybdenum holders, respectively. A normal plate and a shear piezo are mounted on the cantilever holder which can vibrate the cantilevers in normal and lateral directions, respectively.

The microscope employs a sensitive fibre optic interferometer [12] for high force resolution. Sub-Angstrom oscillation amplitudes can be used for imaging as well as performing direct lateral force-distance spectroscopy. Two separate shear-piezo driven 5axis nanopositioners are used to align the fiber with respect to the cantilever. The one is used for the normal force and the other is used for the lateral force measurement. We plan to use L-shaped cantilevers in future to detect the normal and lateral forces simultaneously. However, we concentrated on detection of lateral forces with cantilevers which are only free to oscillate in the lateral direction, as we regulated the tip-sample distance using STM feedback. Straight, etched, home-made tungsten levers from tungsten ribbons are used for this purpose. The operation of the sliders and the alignment of the lateral fiber nanopositioner are shown in Fig. 2. Details of the instrument are described elsewhere [16].

An AFM controller developed by NanoMagnetics Instruments Ltd. [17] is used to control the system. A special radio frequency (RF) circuit is used to inject RF current into the laser diode to improve the interferometer sensitivity. An order of magnitude improvement can be achieved by injecting RF current into the laser. The frequency and the amplitude of the RF current can be adjusted in the software to optimize the noise reduction level. A deflection noise level of $\sim 1 \times 10^{-4} \AA / \mathrm{Hz}^{1 / 2}$ is routinely obtained using this technique. Straight, etched, home-made tungsten levers from tungsten ribbons with a typical stiffness of $50 \mathrm{~N} / \mathrm{m}$ are used in the experiment. The cantilevers are only free to oscillate in the

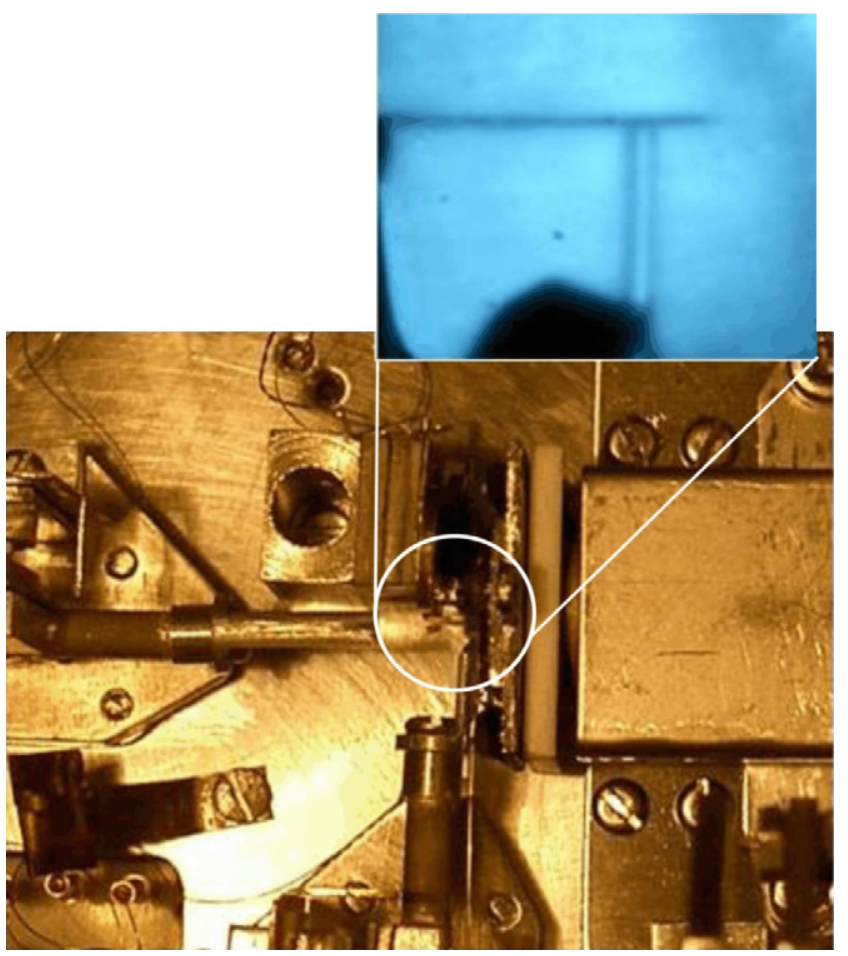

Fig. 2. A picture of the optical fibre aligned at the side of the special lateral force cantilever to measure the lever deflections. 
lateral direction. The stiffness values are calculated from the dimensions of the levers. The vertical detection limit of the microscope is better than $0.1 \AA$, using STM feedback.

$\mathrm{Si}\left(\begin{array}{lll}1 & 1 & 1\end{array}\right)$ sample was cut from $525 \mu \mathrm{m}$ thick, P-doped, n-type wafers oriented within $0.5^{\circ}$ off ( $\left.\begin{array}{lll}1 & 1 & 1\end{array}\right)$ plane. Both ex situ and in situ processes are applied to clean the samples. The sample was cleaned with isopropanol in ultrasonic bath and then rinsed with deionised water. They are then dried with dry nitrogen gas before transferring into the load-lock chamber. The sample is then transferred into the UHV system and degassed at $650{ }^{\circ} \mathrm{C}$ for around $7 \mathrm{~h}$ while the base pressure is kept below $5 \times 10^{-10}$ mbar. Finally the sample is flashed around $1200{ }^{\circ} \mathrm{C}$ for $1 \mathrm{~min}$ and the sample temperature is slowly reduced by means of decreasing the e-beam heater current. The UHV system, which houses the microscope has a base pressure of $7 \times 10^{-11} \mathrm{mbar}$ is pumped with a $300 \mathrm{l} / \mathrm{s}$ Starcell ion pump and Titanium sublimation pump. The system is also equipped with a Turbo-pumped load-lock and a reverse view LEED system.

Fig. 3 shows the operation of the lateral force microscope: the lever is vibrated with sub-Angstrom oscillation amplitudes parallel to the sample surface at a frequency well below its resonance frequency and the changes in the oscillation amplitude and the phase are measured using a lock-in amplifier, while the sample is scanned across the tip. The microscope is operated with STM feedback and simultaneous scans of STM topography, tunnelling current and force gradient can be acquired. The use of very small oscillation amplitudes at frequencies far below resonance allowed us to treat the cantilever motion as a linear spring, and by solving the linearised equation of motion [18], the lateral force gradient between tip and sample can be deduced using simple expression:

$k_{\text {lateral }}=-\frac{d F_{\text {lateral }}}{d x}=k_{0}\left(\frac{A_{0}}{A_{\text {int. }}} \cos \phi-1\right)$

where $k_{\text {lateral }}, k_{0}, A_{0}$ and $A_{\text {int }}$ are interaction stiffness, cantilever stiffness, lateral free oscillation amplitude and the measured lateral amplitude of the cantilever, respectively. $\phi$ is the phase difference between the drive and the lever. The phase difference, $\phi$, between the driving signal and the actual lever motion gives a

(a)

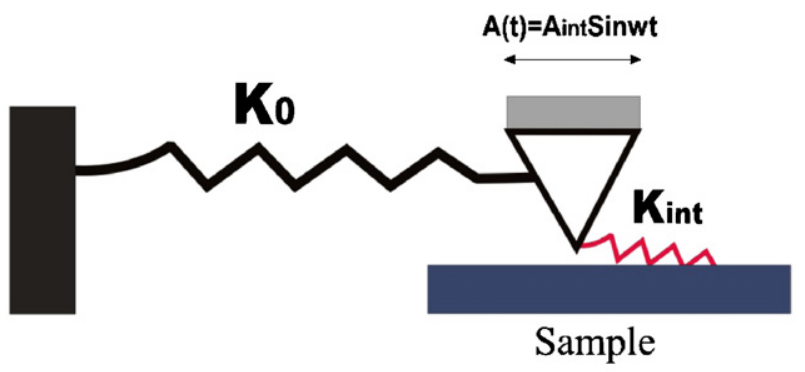

$\sqrt{\eta}$

(b)

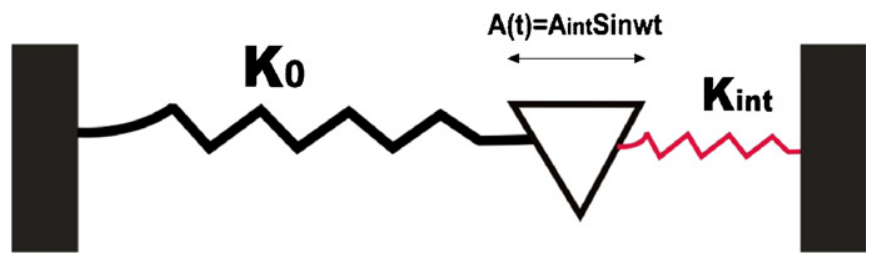

Fig. 3. (a) Schematic view of lateral tip-sample interaction, and (b) mass-spring modelling of the tip-sample interaction. measure of energy losses in the system and hence the friction. We typically measure phase differences less than $6^{\circ}$, which is small enough to neglect the energy loss.

\section{Results and discussions}

In the first series of experiments, we imaged a clean $\operatorname{Si}\left(\begin{array}{lll}1 & 1 & 1\end{array}\right)$ surface using a lateral cantilever.

The cantilever's resonance frequency was found to be $18.049 \mathrm{kHz}$ and the lever was oscillated parallel to the surface with an oscillation frequency of $7.56 \mathrm{kHz}$, far below its resonance frequency with oscillation amplitude of $0.4 \AA_{p}$. The tunnel current is used for feedback to control the tip-sample distance. The scan speed was set to $40 \AA / s$ and the tip bias voltage and set tunnel current were $-1 \mathrm{~V}$ and $0.4 \mathrm{nA}$, respectively. Single and multi 3-atom steps on $\mathrm{Si}\left(\begin{array}{lll}1 & 1 & 1\end{array}\right)$ are resolved in both topography and lateral stiffness channel, as shown in Fig. 3. The lateral stiffness is quantitatively measured. At the two upper terraces, almost zero lateral force gradient is measured. The force gradient has observed to increase by $2.5 \pm 0.65 \mathrm{~N} / \mathrm{m}$ at the single atomic step edge. Very small lateral gradient while the tip scans the upper terraces can be attributed to symmetrical nature of the force gradient and lack of nearby high steps, which the tip apex senses while it is located on the terraces. However, the lateral stiffness increase of approximately $13 \pm 3.5 \mathrm{~N} / \mathrm{m}$ is measured at the lower terrace, at the 3-atom step. This can be due to the existence of 3-atom top high step nearby. It should be noted, the measurement of interaction stiffness varies depend on the location on cantilever side where the fibre is aligned with respect to the tip apex. Due to difficulties to measure the distance precisely, we approximate the effective stiffness within about $25 \%$ variation. The difference of the force gradient at different terraces might be due to high density of defects and impurities on lower terrace, which were not resolved in topography image. The lever stiffness is calculated to be $70 \mathrm{~N} / \mathrm{m}$. It should be noted that the residual noise in the measurements are due to the low oscillation amplitude, as well as the relatively short time constant used in the lock-in amplifier. The image quality can be improved by using a cantilever with high resonance frequency, which would allow us to set higher excitation frequency.

We have also performed lateral force gradient-distance spectroscopy. The feedback loop is frozen, the sample is first retracted back by a specified distance and re-approached towards the tip, while recording the force gradient as well as the tunnel current to perform the spectroscopy. A threshold tunnel current level is used to stop the process and retract the sample. The lever stiffness is calculated to be $70 \mathrm{~N} / \mathrm{m}$ and oscillation amplitude was set to $0.4 \AA_{\text {p. }}$. Fig. 4 shows a force gradient-distance spectroscopy curve obtained on $\mathrm{Si}\left(\begin{array}{lll}1 & 1 & 1\end{array}\right)$ sample. Both the tunnel current and the lateral force gradient increases at the same time. However this behaviour is not typical. We have observed the tunnel current to start increasing before the lateral force gradient in general as demonstrated by Heinrichs [19] and Buhl [20]. The tunnelling barrier height is calculated to be $3.6 \pm 0.3 \mathrm{eV}$ from Fig. 4, which implies that tip and sample are both clean. The effective barrier height is calculated from $I_{\mathrm{t}}(z)=I_{0} e^{(-2 \sqrt{2 m \phi} / h) z}$, where $m$ is the mass of the electron, $h$ is Plank's constant, $\phi$ is the effective barrier height and $z$ is the tip-sample separation. $I_{0}$ is a function of the applied voltage, and the pure barrier height could be slightly bigger. The maximum repulsive force gradient is measured to be $18 \mathrm{~N} / \mathrm{m}$. It should be noted that for both experiments we used the same cantilever, but different sites of the sample and the same bias voltage are used for both measurement, $V_{\text {Bias }}=-1 \mathrm{~V}$. Our measurements reveal that there are significant lateral forces acting on the tip during typical STM experiments. The lateral force gradient at $1 \mathrm{nA}$ tunnel currents can be as large as $10-40 \mathrm{~N} / \mathrm{m}$ depending on the tip shape. The current 

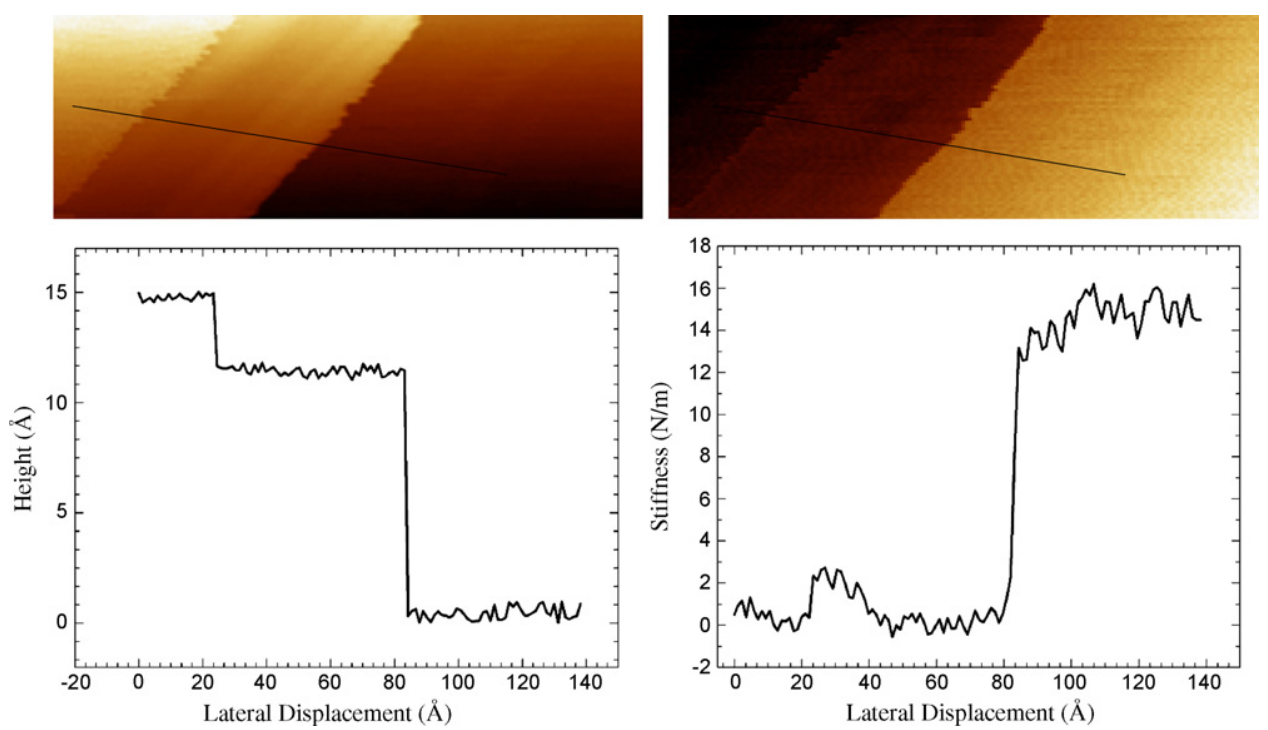

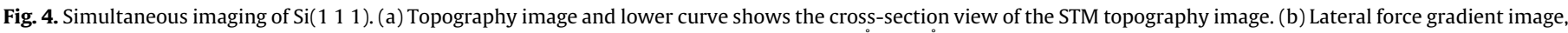

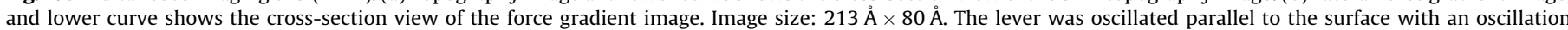
frequency of $7.56 \mathrm{kHz}$ and oscillation amplitude of $0.4 \AA_{\mathrm{p}}$. Tip bias voltage and set tunnel current were $-1 \mathrm{~V}$ and $0.4 \mathrm{nA}$, respectively.

levels tested here are typical currents that are used at STM experiments (Fig. 5).

In conclusion, a novel fibre optic interferometer based lateral nc-AFM/STM is used to investigate the lateral force gradients between tungsten tip and the $\operatorname{Si}\left(\begin{array}{lll}1 & 1 & 1\end{array}\right)-(7 \times 7)$ surface. The improvement in the resolution of the interferometer allowed us to use very small oscillation amplitudes to oscillate the cantilever parallel to the surface, which overcomes the problems associated with the large amplitude technique that has been widely used. Since we use small amplitudes, we can extract lateral forces with unprecedented sensitivity by measuring the changes in the oscillation amplitude directly, rather than the frequency shift. We have observed single and multi atomic steps in topography and lateral stiffness images. This information allowed us to measure the lateral force direct and quantitatively. Our experiments reveal the first direct measurement of lateral stiffness in dynamic mode AFM. In contrast to other indirect experiments, where local formal force-distance curves are used to calculate lateral force gradients [11], our method offers direct and

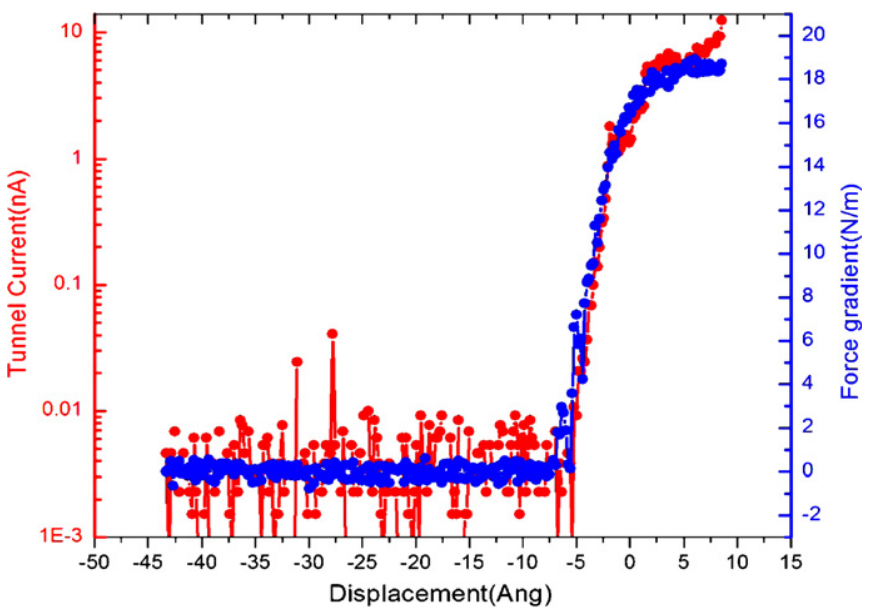

Fig. 5. Simultaneous lateral force gradient-distance and tunnel current vs. distance spectroscopy. The sample bias voltage was set on $-1 \mathrm{~V}$. The cantilever free oscillation amplitude $0.4 \AA_{\mathrm{p}}$. independent measurements of lateral force gradients. Lateral force gradient-distance spectroscopy experiments have also been carried out, in which we simultaneously measured the force gradient, and tunnelling current as the sample is approached towards the tip and retracted back. We obtained lateral force gradient-distance curves exhibiting sharp increase of the force gradient, while the sample is approaching to the surface. We observed positive and sometimes slightly negative force gradients. We attribute the lack of pronounced attractive region in the lateral force gradient could be due to cancellation of long range attractive forces acting on the tip because of the symmetry of the surface structure. We observed for the first time, surprisingly large, lateral stiffness in typical STM operating conditions, $\sim 1 \mathrm{nA}$.

\section{Acknowledgments}

This project is funded in Turkey by TÜBITAK Grant No. TBAG2329 and the European Union NANOMAN Grant from FP6 Framework Program. We would like to thank Mr. Muharrem Demir of NanoMagnetics Instruments Ltd. for the technical support. A.O. thanks Turkish Academy of Sciences (TÜBA) for partial financial support.

\section{References}

[1] B.N.J. Persson, Sliding Friction, Physical Principles and Applications, 2nd edition, Springer, Berlin, 2000.

[2] C.M. Mate, G.M. McCleland, R. Erlandsson, S. Chiang, Phys. Rev. Lett. 59 (1987) 1942.

[3] L. Howald, R. Luthi, E. Meyer, G. Gerth, H. Haefke, R. Overney, H.J. Guentherodt, J. Vac. Sci. Technol. B 12 (1994) 2227.

[4] R. Bennewitz, E. Gnecco, T. Gyalog, E. Meyer, Tribol. Lett. 10 (2001) 51.

[5] S.P. Jarvis, H. Yamada, K. Kobayashi, A. Toda, H. Tokumoto, Appl. Surf. Sci. 157 (2000) 314.

[6] F. Giessibl, M. Herz, J. Manhart, Proc. Natl. Acad. Sci. U.S.A. 99 (2002) 12006

[7] O. Pfeiffer, R. Bennewitz, A. Baratoff, E. Meyer, Phys. Rev. B 65 (2002) 161403.

[8] S. Kawai, S. Kitamura, D. Kobayashi, H. Kawakatsu, Appl. Phys. Lett. 87 (2005) 173105.

[9] A. Schwarz, H. Hölscher, S.M. Langkat, R. Wiesendanger, AIP Conference Proceedings of STM, Vol. 696, 2003, p. 68.

[10] M. Abe, Y. Sugimoto, T. Namikawa, K. Morita, N. Oyabu, S. Morita, Appl. Phys. Lett. 90 (2005) 203130.

[11] Y. Sugimoto, T. Namikawa, K. Miki, M. Abe, S. Morita, Phys. Rev. B. 77 (2008) 195424 
[12] A. Oral, R.A. Grimble, H.Ö. Özer, P.M. Hoffmann, J.B. Pethica, Appl. Phys. Lett. 79 (1915) 2001.

[13] P.M. Hoffmann, A. Oral, R.A. Grimble, H.Ö. Özer, S. Jeffery, J.B. Pethica, Proc. Royal Soc. A 457 (2001) 1161.

[14] H.Ö. Özer, M. Atabak, R.M. Ellialtıŏglu, A. Oral, Appl. Surf. Sci. 188 (2002) 301.

[15] A. Oral, R.A. Grimble, H.Ö. Özer, J.B. Pethica, Rev. Sci. Inst. 84 (2003) 3656.
[16] M. Atabak, A. Oral, (unpublished).

[17] NanoMagnetics Instruments Ltd., Suite 290, 266 Banbury Road, Oxford OX2 7DL, UK, www.nanomagnetics-inst.com.

[18] P.M. Hoffmann, Appl. Surf. Sci. 210 (2003) 140.

[19] J. Heinrichs, Solid State Commun. 13 (1973) 1595.

[20] W. Buhl, Z. Physik B 23 (1976) 221. 